\title{
Observing shadow of the Schwarzschild black hole in presence of a plasma
}

\author{
Farruh Atamurotov \\ National University of Uzbekistan, Tashkent 100174, Uzbekistan \\ Ulugh Beg Astronomical Institute, Astronomicheskaya 33, Tashkent 100052, Uzbekistan \\ Inha University in Tashkent, Tashkent 100170, Uzbekistan \\ email: atamurotov@yahoo.com
}

\begin{abstract}
We have investigated particle motion around Schwarzschild black holes in the presence of a plasma with radial power-law density profile has been shown that the photon sphere around a spherically symmetric black hole is unchanged under the influence of the plasma; however, the Schwarzschild black hole shadow size is reduced due to the refraction of electromagnetic radiation in the plasma environment of the black hole.
\end{abstract}

\section{Introduction}

The study of astrophysical processes in the plasma medium surrounding a black hole becomes very interesting and important due to the evidence for the presence of black holes at the centres of the galaxies. For example, the gravitational lensing in inhomogeneous and homogeneous plasma around black holes has been recently studied in BisnovatyiKogan and Tsupko (2010) as an extension of vacuum studies.

The shape of the black hole is determined through the boundary of the shadow which can be studied by application of the null geodesic equations in Atamurotov et al. (2016). The presence of a plasma in the vicinity of black holes changes the equations of motion of photons which may lead to the modification of the black hole shadow. In this paper our goal is to consider the silhouette of the shadow of an axially symmetric black hole using the equations of motion for photons in a plasma with radial power-law density profile. We would like to underline that recently, the influence of a non-magnetized cold plasma with the radially dependent density to black hole shadow has been studied by Perlick et al. (2015) using a different approach.

\section{Photon motion around kerr black hole}

The rotating black hole is described by the spacetime metric, which in the standard Boyer-Lindquist coordinates can be written in the form

$$
\begin{aligned}
d s^{2}= & -\left(1-\frac{2 M r}{\Sigma^{2}}\right) d t^{2}+\frac{\Sigma^{2}}{\Delta} d r^{2}+ \\
& \Sigma^{2} d \theta^{2}-\frac{4 a M r \sin ^{2} \theta}{\Sigma^{2}} d t d \phi+ \\
& \sin ^{2} \theta\left[\Sigma^{2}+\frac{a^{2}\left(\Sigma^{2}+2 M r\right) \sin ^{2} \theta}{\Sigma^{2}}\right] d \phi^{2},
\end{aligned}
$$

where

$$
\begin{aligned}
\Sigma^{2} & =r^{2}+a^{2} \cos ^{2} \theta, \\
\Delta & =r^{2}-2 M r+a^{2} .
\end{aligned}
$$




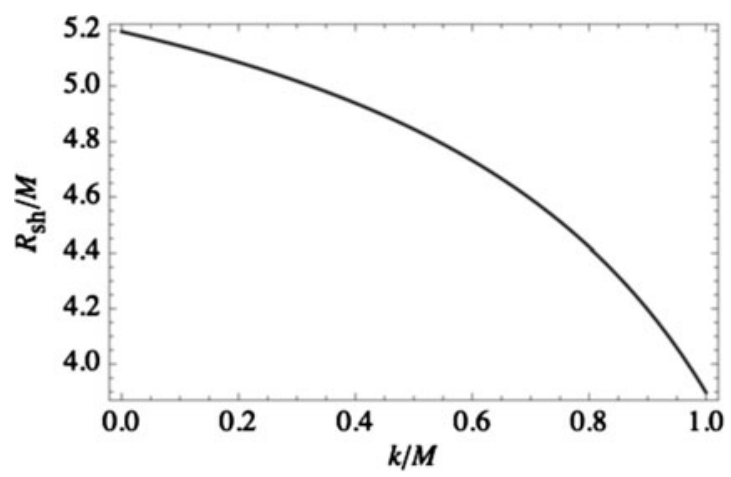

Figure 1. The dependence of the Schwarzschild black hole shadow on the plasma frequency parameter in Atamurotov et al. (2015)

Using the celestial coordinates one can easily describe the shadow in Vázquez and Esteban (2004), Atamurotov (2016):

$$
\alpha=\lim _{r_{0} \rightarrow \infty}\left(-r_{0}^{2} \sin \theta_{0} \frac{d \phi}{d r}\right),
$$

and

$$
\beta=\lim _{r_{0} \rightarrow \infty} r_{0}^{2} \frac{d \theta}{d r}
$$

In Fig. 1, shows that the radius of the shadow of black hole surrounded by an inhomogeneous plasma decreases in Atamurotov et al. (2015).

\section{Conclusion}

In order to extract the pure effect of the influence of a plasma on the black hole image, the particular case of the Schwarzschild black hole has also been investigated. It was shown that under the influence of a plasma, the observed size of the shadow of the spherically symmetric black hole becomes smaller than that in the vacuum case due to the refraction of the electromagnetic radiation in the plasma.

\section{References}

Bisnovatyi-Kogan, G. S. \& Tsupko, O. Yu. (2010), Mon. Not. R. Astron. Soc., 404, 1790. Perlick, V., Tsupko, O. Yu. \& Bisnovatyi-Kogan, G. S. (2015) Phys. Rev. D, 404, 1790. Atamurotov, F., Ahmedov, B., \& Ghosh, S. G. (2016) Eur. Phys. J. C, 76, 273. Atamurotov, F. (2016) Proceedings of the International Astronomical Union, IAU Symposium, 312, pp. 135-136.

Atamurotov, F., Ahmedov, B., \& Abdujabbarov, A. (2015) Phys. Rev. D, 92, 084005.

Vázquez S. \& Esteban, E. (2004) Nuovo Cim. 119B, 489. 